\title{
DESIGN OF A POWERFUL AND PORTABLE MULTIDIRECTIONAL WAVEMAKER
}

\begin{abstract}
Andrew Cornett ${ }^{1}$, Peter Laurich ${ }^{2}$, Enrique Gardeta ${ }^{3}$, Daniel Pelletier ${ }^{4}$
A new multidirectional wave generator with 72 independent paddles has been designed, fabricated and commissioned at the National Research Council labs in Ottawa, Canada. The wet-back piston-mode machine is installed in a new $50 \mathrm{~m}$ long by $30 \mathrm{~m}$ wide rectangular wave basin, where water depths can be varied over the range from $0 \mathrm{~m}$ up to $1.3 \mathrm{~m}$. The new machine is believed to be unique in the world in that it combines the power and stroke required to generate multidirectional spectral wave conditions with significant wave heights exceeding $0.4 \mathrm{~m}$ together with the modularity and ease of portability required to move the machine quickly and safely to new positions. The new machine can also be sub-divided to form several shorter machines if desired. The new wave generator features lightweight, composite materials, energy efficient regenerative power supplies, state-of-the-art software and control systems, including capabilities for active wave absorption (reflection compensation), second-order wave generation for improved generation of nonlinear sub- and super-harmonics, side-wall reflection, and more. The design of this new directional wavemaker is described and several of the more innovative features are highlighted in this paper.
\end{abstract}

Keywords: wave generator, wavemaker, multidirectional waves, control system, physical modeling

\section{INTRODUCTION}

Physical model studies of coastal processes, coastal and port structures, marine vehicles and marine operations depend on the ability to reproduce a wide range of realistic wave conditions within test facilities. Unfortunately, the quality and usefulness of such studies are often limited by the capabilities of available wave generation equipment. Many wavemakers can generate only small amplitude waves, which forces the engineer / researcher to work at a small scale when simulating storm conditions. This limitation has serious consequences, since scale effects related to distortions in Reynolds number and surface tension become increasingly important in small scale models. Moreover, many wave generation systems are unable to generate multidirectional (short-crested) waves or correctly reproduce infra-gravity (low frequency) waves or compensate for waves reflected from a test structure. All of these deficiencies can seriously degrade the quality of physical model studies of processes dominated by wave action, bringing physical model study results into question. These limitations increase the risk of failure for real structures whose designs were confirmed in physical model studies. New machines with improved capabilities for reproducing realistic wave conditions in test facilities at large scale can make important contributions to the quality of coastal engineering research and practice.

This paper summarizes the design and fabrication of a new multidirectional wave generator that includes many essential features required to maximize the quality of physical model experiments and studies involving waves. The new wet-back type wavemaker (see Figure 1) has been designed to have the power and stroke required to generate short-crested and long-crested irregular waves with significant wave heights exceeding $0.4 \mathrm{~m}$, as well as regular waves with heights over $0.65 \mathrm{~m}$, sufficient to simulate $9 \mathrm{~m}$ high waves at a length scale of 1:15. It also features state-of-the-art software and control systems, including capabilities for active wave absorption (reflection compensation), secondorder wave generation (for improved generation of nonlinear sub- and super-harmonics), side-wall reflection, and more. Moreover, the new wave generator features a highly modular design and is fully portable. The stiffness to weight ratio of several key components, including the wave paddles, has been maximized through extensive use of high strength aluminum and composite materials, while power consumption has been minimized by incorporating energy efficient regenerative power supplies. Each module supports eight wave paddles moving in piston-mode, and the modules can be arranged in various configurations to support project requirements. For example, the modules can be arranged to form one long wave generator or several shorter wavemakers. This innovative and capable new machine has been developed for the National Research Council of Canada (NRC) by Akamina Technologies (Canada) in partnership with +D (Spain) with support from Respect Industries (Canada), Bosch-Rexroth (Canada), IH Cantabria (Spain) and M.D. Miles (Canada).

The new wave generator has been developed for use within a new $50 \mathrm{~m}$ by $30 \mathrm{~m}$ rectangular wave basin at the NRC's Ocean, Coastal and River Engineering research lab in Ottawa, Canada. Nine modules each with eight wave paddles have been fabricated to date. The new wave generator has been

\footnotetext{
${ }^{1}$ Ocean, Coastal \& River Engineering, National Research Council Canada, Ottawa, Ontario, Canada

${ }^{2}$ Akamina Technologies, Ottawa, Ontario, Canada

${ }^{3}+\mathrm{D}$, Madrid, Spain

${ }^{4}$ Ocean, Coastal \& River Engineering, National Research Council Canada, Ottawa, Ontario, Canada
} 
successfully commissioned and is being used to generate realistic wave conditions in physical model experiments and studies.

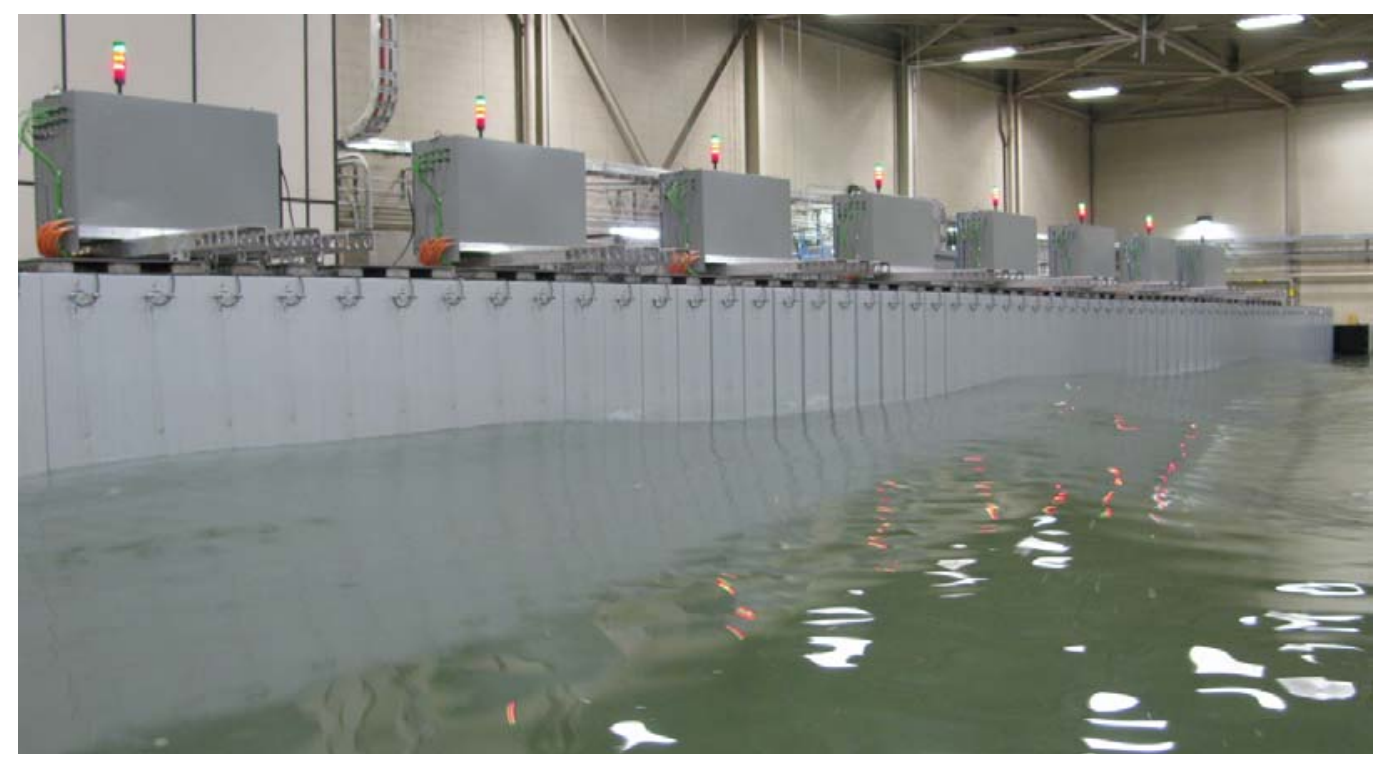

Figure 1. New 72-segment portable directional wave generator.

\section{SPECIFICATIONS}

The new wave generator has been designed to satisfy a long list of specifications and performance requirements, including those listed below.

- The system must be a wet-back piston-type segmented directional wave generator.

- The system must include no less than 72 wave paddles (segments).

- The wave paddle width must be within $0.4 \mathrm{~m}$ to $0.45 \mathrm{~m}$.

- The wave paddle height must be no less than $1.4 \mathrm{~m}$.

- The gap between adjacent wave paddles must be no more than $5 \mathrm{~mm}$.

- The minimum useful wave paddle stroke shall be at least $1.0 \mathrm{~m}$.

- The system must be able to generate the full range of regular wave conditions shown in Figure 2.

- The system must be modular in construction and the maximum weight of each module must not exceed 2,350 kg.

- The wave generator must be portable. It shall be possible for two technicians to move the entire wave generator to a new location and secure it within a single 7.5 hour workday.

- Lifting points shall be provided to facility safe and efficient movement of the modules by forklift.

- It must be possible to arrange the modules to form one continuous wave generator or two shorter wave generators in two locations. The ability to operate the two shorter generators individually or simultaneously is required.

- For stability during wave generation, the design must support a) placing ballasting weights on top of the modules and/or b) securing the modules to the concrete floor.

- The system must employ AC servo motor drive systems capable of operating at velocities between 0 up to $0.9 \mathrm{~m} / \mathrm{s}$.

- The maximum peak power consumption of the system shall be less than 750 kVA @ 460 Volts 3 Phase.

- The system must include redundant safety features to minimize the risk of damage and accidental injury.

- The system must support the synthesis and generation of regular waves, irregular unidirectional waves (normal and oblique) and irregular multidirectional waves.

- The system must support active wave absorption, side-wall reflection and the synthesis and generation of waves consistent with second-order wave theory.

- The design life of the system must exceed 20 years, assuming operation for 500 hours/year. 


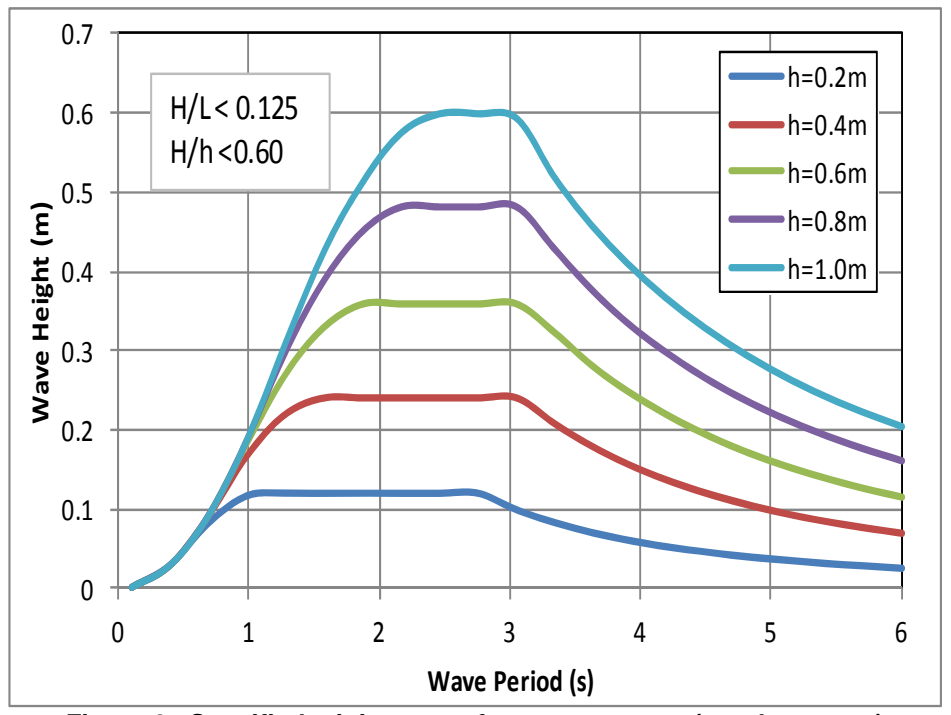

Figure 2. Specified minimum performance curves (regular waves)

\section{MECHANICAL DESIGN}

The new wave generator uses a modular design in which the individual modules to be arranged in various configurations to support diverse project requirements. The basic module consists of 8 electrically actuated wave paddles supported on a rigid stainless steel frame (see Figure 3). Nine modules, each supporting eight wave paddles have been fabricated so far. Each of the $0.41 \mathrm{~m}$ wide by $1.7 \mathrm{~m}$ tall wave paddles is driven by a synchronous AC servo-motor connected to a ball screw via a backlash-free coupling. The servo drives for the motors, the control computer and all internal cabling is enclosed in a IP65 servo drive cabinet mounted on the top of the module. The number of cables feeding into each servo drive cabinet has been minimized to make it easier and quicker to rearrange the wave generator modules into various configurations. Several modules can be joined together using specially designed clamps to form integrated wavemakers of various lengths. The nine modules built to date can be arranged in various configurations: to form a single $29.5 \mathrm{~m}$ long wave machine (with 72 paddles) at any location in the $50 \mathrm{~m}$ by $30 \mathrm{~m}$ basin; to form an "L" configuration along two adjacent walls; or even to form two independent wave machines with a combined length of $29.5 \mathrm{~m}$.

To ensure precise control over wave paddle motion, the wave paddles and support frames have been designed to have extremely high rigidity while the drivetrains feature a backlash-free ball-screw that converts rotational motion to very precise linear motion. Each wave paddle has a working stroke of $\pm 0.55 \mathrm{~m}$ and a rated maximum velocity of $1.0 \mathrm{~m} / \mathrm{s}$, and can generate peak forces over $2.5 \mathrm{kN}$, sufficient to generate regular waves with heights exceeding $0.6 \mathrm{~m}$ and waves with periods from $0.5 \mathrm{~s}$ to $5 \mathrm{~s}$, with considerable reserve capacity for active wave absorption (reflection compensation). The total weight of each module was kept below 2,350 kg to ensure that it could be moved easily within the basin using only a forklift or hydraulic lift truck. To ensure stability when generating large waves, the modules can either be fastened to the basin floor using specially designed clamps, or stabilized by placing ballasting weights on top of the sturdy steel support frame. No anchoring or ballasting is required when generating smaller waves.

Each wave generator module has four levels as shown in Figure 3 (see also Figure 4a).

The floor level. The levelling feet and the lower module integrated connection clamps are located in this lower level. The feet are equipped with high-friction pads, a ball joint and vertical adjustment to account for unevenness in the floor. 


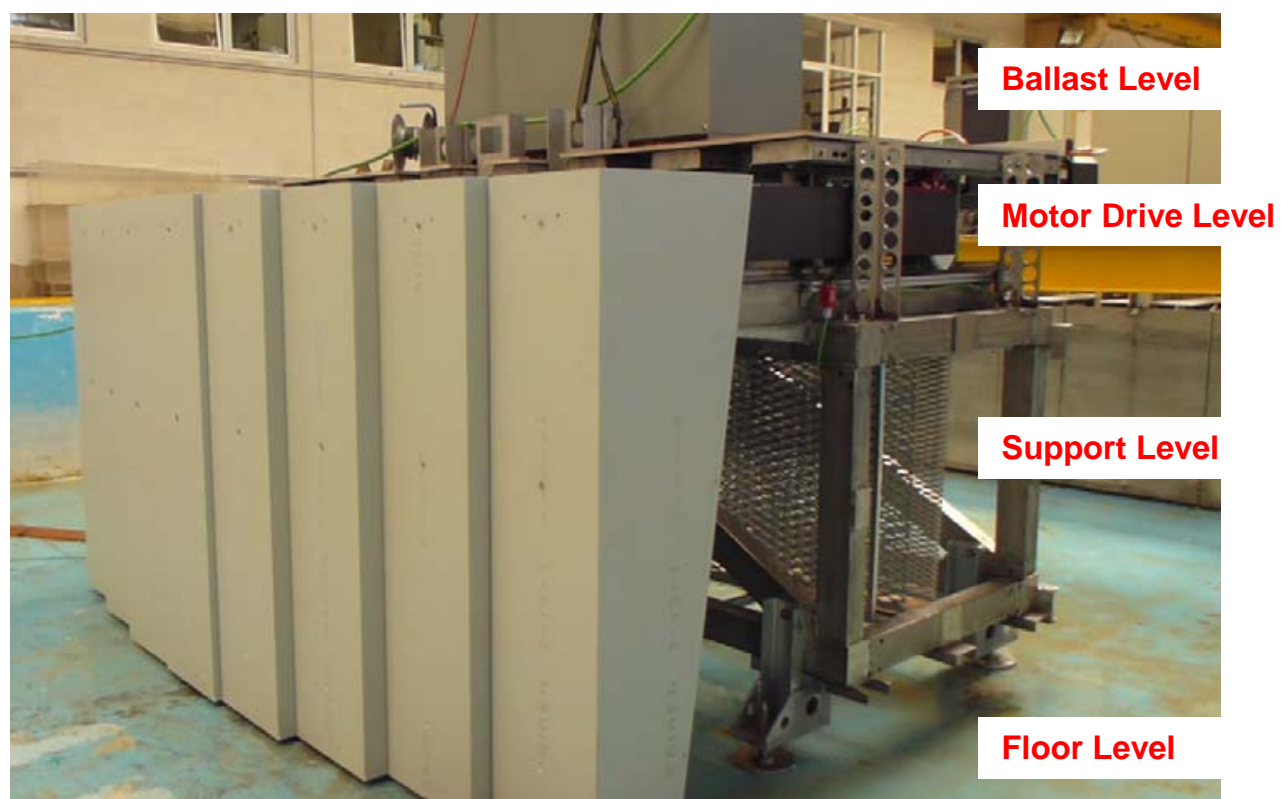

Figure 3. Each wave generator module has four distinct levels and supports eight $0.41 \mathrm{~m}$ wide by $1.7 \mathrm{~m}$ tall wave paddles.

The support level. This is the main structure that supports the upper levels including the powertrain and wave paddle main beam guiding system, while providing overall strength and rigidity. The main frame (see Figure 4b) is made entirely of stainless steel and designed for high strength and high rigidity with negligible corrosion. The steel frame includes lateral locating fixtures to facilitate rapid assembly and clamping of several adjacent modules into a single wave generator unit. The support frame is fitted with expanded perforated metal sheets which serve to dissipate wave energy behind the wave paddles.

The motor drive level. The servo motors, lead screws and backlash-free transmissions are located on this level (see Figure 4d). Each wave paddle is powered by a Bosch Rexroth IndraDyn S MSK061C synchronous servo motor acting through the backlash-free transmission. The MSK family features a wide power spectrum, high torque density, a range of encoder options, brake options and IP65 protection. The selected motor has a maximum speed of 6,000 RPM, a continuous torque of $12 \mathrm{Nm}$ and a maximum torque of $32 \mathrm{Nm}$. The wave paddles themselves are comprised of aluminum sandwich panels offering very low weight together with extremely high rigidity and dimensional stability. Additional stiffness is provided by an inner "skeleton" structure, also in high strength anodised aluminum alloy (see Figure 4c). The wave paddle design represents an ideal balance between high stiffness, light weight and durability. Each wave paddle is rigidly bonded to a lightweight and rigid anodized aluminium main box beam that travels horizontally on a monorail guidance system (see Figure 4e). The linear guide rail and ball-bearing carriages are coated to withstand continuous splashing, although no wetting is expected under normal operating conditions.

Both the wave paddle and its sub-structure are exclusively built in lightweight materials (aluminum alloy and composites). High rigidity with low weight has been achieved by applying a design philosophy based around structural box sections and by using aerospace construction techniques. The design achieves a very high ratio of rigidity/strength to weight that delivers improved frequency response and requires less energy to overcome machine inertia and friction, leading to improved precision control of wave paddle movement. The lightweight wave paddles can be easily removed (7 screws) for maintenance, or to avoid accidental contact when maneuvering adjacent modules into position beside each other. Each wave paddle is fitted with a capacitance-wire wave gauge to sense the local water level; information required to support active wave absorption (reflection compensation).

The ballast level. Ballast weights made of lead, steel or concrete can be placed on top of the module for added stability during wave generation. The forklift lift points and the electrical cabinet containing the servo-drives and distributed control computer are also located on this upper level together with an accessible marine plywood service deck. 
a)

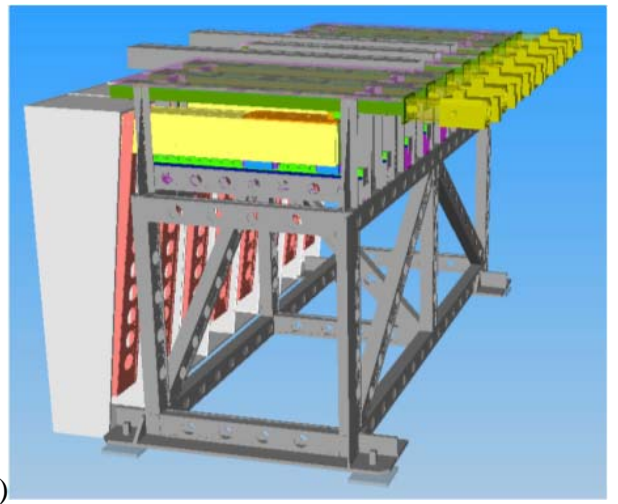

b)

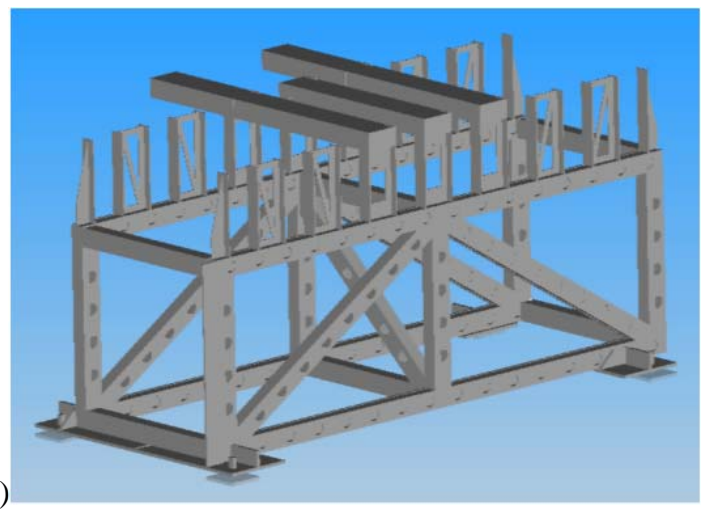

d)
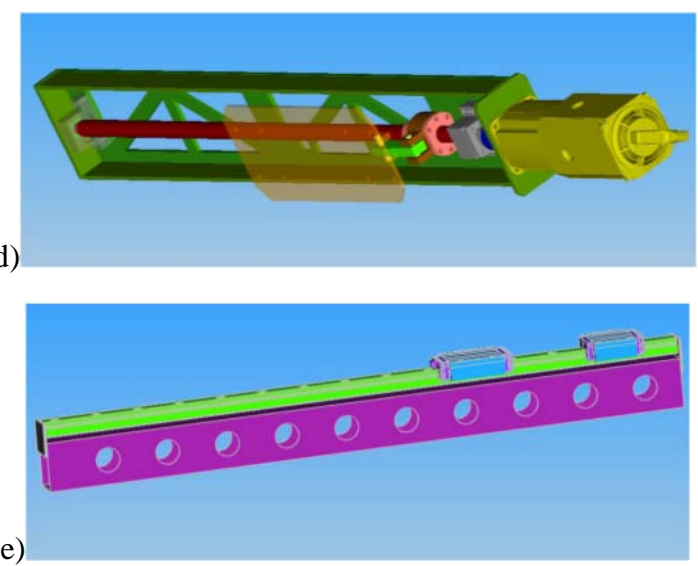

e)

Figure 4. 3D CAD modelling: a) module assembly; b) stainless steel frame; c) wave paddle sub-assembly; d) powertrain sub-assembly; e) monorail guide system sub-assembly.

\section{CONTROL SYSTEM DESIGN}

Figure 5 shows a high-level top-down view of the wave generation system. A brief description of each of the main components is given below.

Operator's Console - a PC that runs the interface to the wave generation system. Additional Operator's Consoles can be added to support situations in which the models are grouped to form two or more separate wave generators.

Remote Control Console - a rack-mounted console that provides basic visual status information and for managing basic operations. Status information such as power on / off indications and control interlock loop state are visible on the console. The operator can turn on and off AC power, reset the control system and turn on and off the high voltage DC bus that powers the servo drives from the console. There are also network connection points for the Operator's Console as well as connectors for extending the control interlock loop as well as the AC power interlock loop.

3-Phase AC Central Power - transformers and control circuitry required to provide the main AC power to the system. The power to the system is turned on and off from the Remote Control Console.

AC Interlock Loop - one of two independent safety interlock loops in the system. The AC power interlock loop controls the main AC power to the system. The AC power to the servo drive cabinets is immediately cut if the interlock loop is opened using any of a number of red emergency pushbuttons strategically located around the facility.

AC Power Outlets - a series of eighteen AC power outlets distributed along two walls of the basin. Each outlet provides sufficient 3-phase AC power for a single Servo Drive Cabinet.

Servo Drive Cabinet - all of the components required to provide the controlling pulse-width modulation (PWM) power signals to the servo motors on a module of 8 segments are enclosed within a cabinet. One cabinet is mounted on top of each of the mechanical modules. 
Synchronization, Network, Management and Interlock - these signals are combined into a single, multi-drop cable that starts at the Remote Control Console and ends at a terminator after the last Servo Drive Cabinet in the chosen configuration.

Control Interlock Loop - this is the second interlock loop in the system. The control interlock loop is carried in the Synchronization, Network, Management and Interlock Cable. The drives are programmed to execute a motor-assisted stop procedure if this loop is opened. The control interlock loop can be opened using any of a number of yellow emergency pushbuttons located around the facility or by the control system itself.

8-Segment Module - the 8-segment module consists of the stainless steel frame, wave paddles, transmission systems and motor drives for the 8 segments in the module.

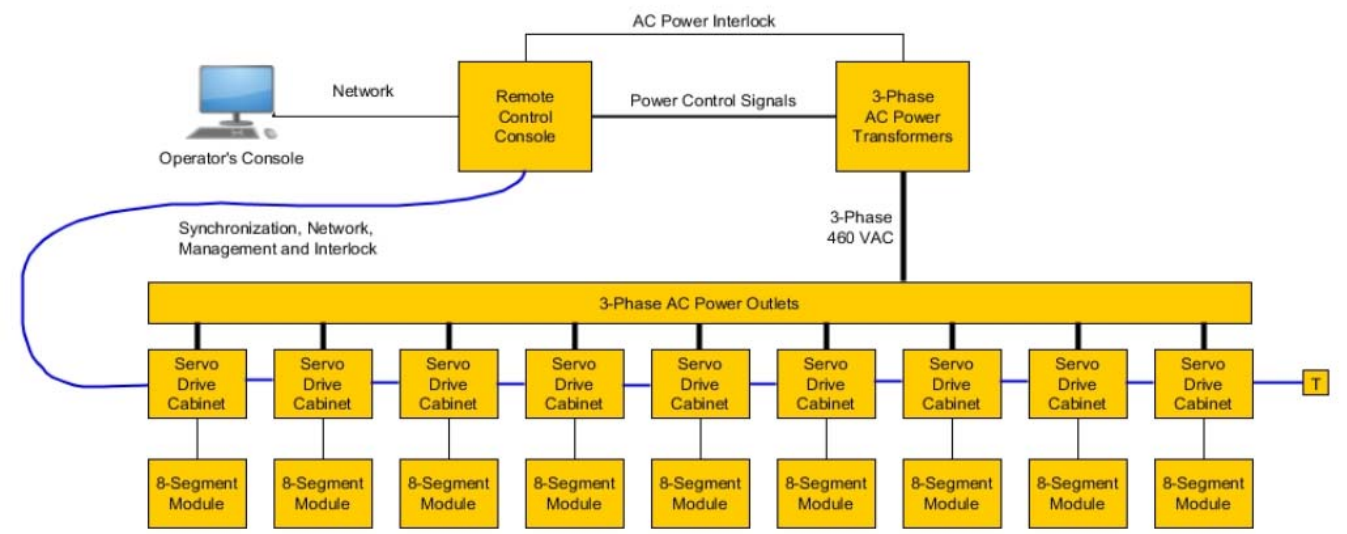

Figure 5. High-level top-down view of the wave generation system.

\section{Operator's Console}

The Operator's Console is a PC running the Windows 7 operating system. The wave generator is operated and controlled through the WaveGen-Studio software developed by Akamina Technologies. WaveGen-Studio is a feature-rich, powerful system designed for managing hydrodynamic experiments and physical model studies. It combines the four components of data acquisition, wave synthesis, wave generation and wave data analysis in a seamless, intuitive graphical interface designed to allow users to easily manage even the most complex experiments. Figure 6 shows a screen shot of the operator's interface during wave generation.

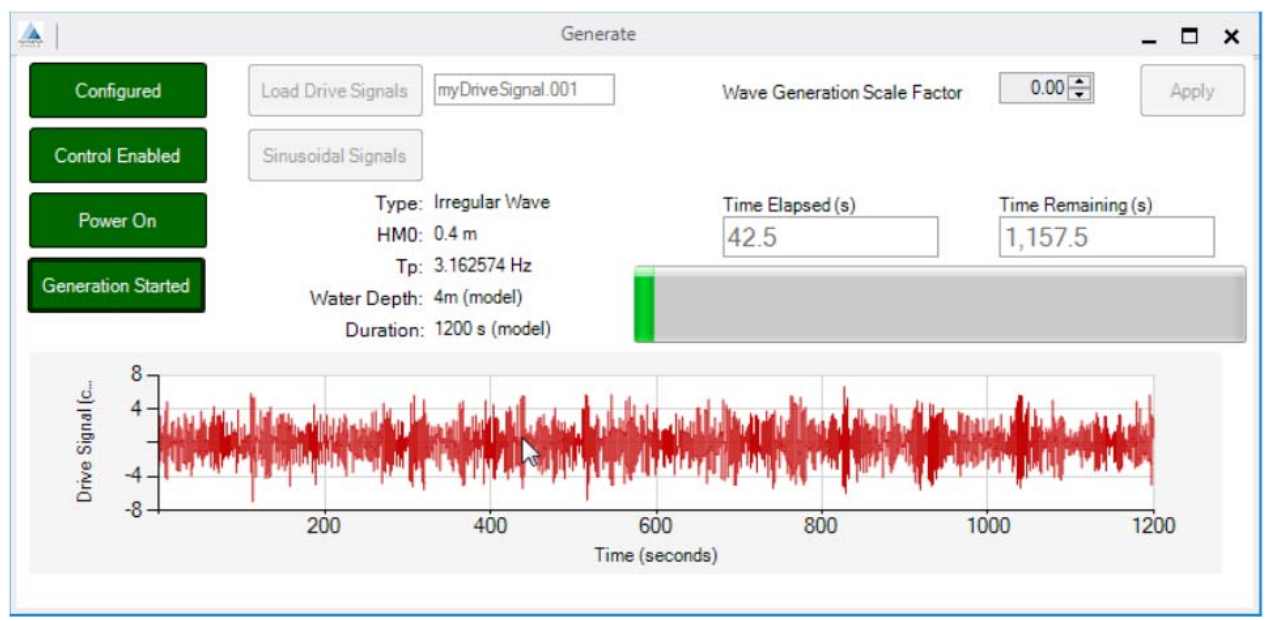

Figure 6. Screen shot of the user interface. 
The wave generation system uses a network of connected controllers to provide the necessary control capabilities. One controller is housed within each of the Servo Drive Cabinets with the controllers and the Operator's Console connected on a Gigabit Ethernet network.

The wave synthesis, wave data analysis and plotting functionality within WaveGen-Studio are based on the GEDAP software system developed over several decades at the National Research Council, Canada (Miles, 1989, 1997). GEDAP is a well-proven, complete software solution for physical modelling in hydrodynamic laboratories. Commonly used GEDAP programs have been integrated into WaveGen-Studio for interactive use. Other GEDAP programs, the GPLOT plotting package, customized scripting and batch file processing are also available through the Windows Command Prompt.

\section{Data Acquisition}

The data acquisition component of WaveGen-Studio allows data to be acquired from the control servers for which the system has been configured. The data acquisition features include:

- Acquire data from any number of control server input channels or derived data channels at the selected sampling frequency.

- Calibrate single channels or groups of channels.

- Display strip-charts with multiple channels per strip-chart in real-time.

\section{Wave Synthesis}

The wave synthesis and wave analysis capabilities of WaveGen-Studio are based on the GEDAP suite of programs developed at the NRC over several decades. Mansard and Miles (2010) provide an overview of the wave generation and analysis capabilities of the GEDAP system. The graphical wave synthesis interface within WaveGen-Studio provides access to the GEDAP 2D and 3D wave synthesis and generation libraries. The interface allows wave spectra to be created, wave height time series and directional spectra to be derived and wave paddle drive signal files to be computed and loaded into controllers for wave generation. Cornett and Miles (1991) discuss the synthesis and reproduction of multidirectional hurricane sea states in a wave basin using a previous version of the GEDAP 3D library. The GEDAP wave generation software has been enhanced and expanded over the years and currently includes the following capabilities, among others:

- $\quad$ synthesis and generation of regular, irregular (long-crested and short-crested) seastates, and episodic waves of various types;

- control over wave grouping characteristics and wave asymmetry factors;

- $\quad$ synthesis according to 2nd order theory for correct generation of bound sub- and super-harmonics;

- $\quad$ support for side-wall reflection based on linear wave theory and a new diffraction theory method;

- $\quad$ support for piston, hinged-flapper and combination modes of wave paddle articulation;

- $\quad$ support for elevated wave generators;

- $\quad$ support of active wave absorption (reflection compensation);

- dynamic calibration of wave generators to improve wave generation fidelity; and

- $\quad$ spectral matching transfer functions to achieve a better match between measured and desired time histories and spectra.

Target wave spectra can be specified using parametric models or by importing measured wave spectra. Parametric models that are supported include: Pierson-Moskowitz, JONSWAP, Bretschneider, Ochi Double-Peak, Scott, TMA Shallow Water, Newmann, Mitsuyasu-Bretschneider, and Mitsuyasu (1972). Wave trains can be synthesized from target wave spectra using the Fast Fourier Transform random phase, random Fourier Coefficient or random Fourier Coefficient with matched variance methods (Mansard and Miles, 2010). The GEDAP wave generation software has proven over decades of use to reliably convert wave spectra to wave height time series and wave height time series to paddle positions with a high degree of accuracy and precision. Moreover, it includes software tools that allow the measured wave spectrum to be used to adjust drive signals to achieve improved agreement with a desired target spectrum.

\section{Wave Generation}

Wave generation is the process of moving one or multiple wave paddles to generate waves. Wave generation with and without active wave absorption are supported. For this facility, a bank of controllers provides closed-loop control of the position or velocity of the single actuator that drives each wave paddle. 


\section{Wave Data Analysis}

The functionality to analyze wave data in a comprehensive manner is provided through a suite of programs designed specifically for the analysis of $2 \mathrm{D}$ and $3 \mathrm{D}$ waves. The data analysis software includes a broad range of analysis tools organized in the following four groups:

- $\quad$ time-domain: tools for zero-crossing analysis, trend removal, peak detection and filtering;

- frequency-domain: tools for wave reflection analysis, variance spectral density analysis, filtering, differentiation and integration;

- $\quad$ statistics: tools for basic and advanced statistics, probability distributions, and wave-by-wave statistics; and

- $\quad$ miscellaneous: tools for data scaling, sub-record selection, resampling, importing and exporting.

Directional wave spectra can be computed using either the Maximum Likelihood Method or the Maximum Entropy Method (Nwogu, et al., 1987). Support for directional analysis of data from wave gauge arrays as well as co-located measurements of free surface oscillations and orbital velocities or surface slopes is provided (Cornett, et al., 1995).

\section{Servo Drive Cabinet}

The Servo Drive Cabinet (SDC) is the heart of the power and control for the wave generators. One of the important design features of the cabinet is that it uses regenerative components to reduce power consumption. When the motors decelerate, the inertia of the load causes the motor to act as a generator. The standard procedure for dealing with this generated power is to short it to ground through high current resistors. Given the rising cost of electricity and the substantial amount of energy consumed by wave generation systems, the power system was designed to take the generated energy and return it to the grid rather than waste it as heat. The potential energy savings and the more responsible use of energy are a significant feature of the new wave generation system. The arrangement of components within the cabinet is shown below in Figure 7.

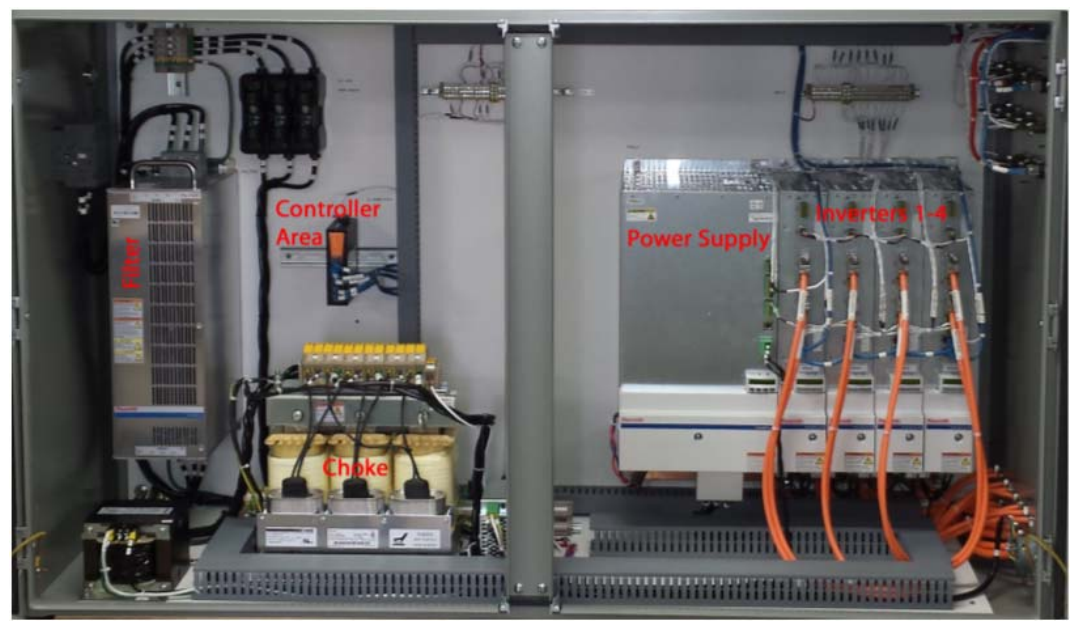

Figure 7. Arrangement of components within a Servo Drive Cabinet.

\section{Power Supply (PSU)}

The system uses a Bosch Rexroth HMV01 regenerative power supply with a rated power of $45 \mathrm{~kW}$. The regenerative power capability allows the system to recover power that would otherwise be dissipated as heat when the wave paddles are being decelerated.

\section{Inverter (Servo Drive)}

The servo drive is the Bosch Rexroth IndraDrive Cs model HMD01 dual-axis drive with a CDB02 control card.

\section{Controller}

The controller provides high-level management of the servo drives. It acquires real-time feedback from the drives from which it computes the control value that is then sent to each drive. Wave paddle position and velocity feedback is derived from angular encoders integrated into each drive, while the capacitance wave gauges mounted on the face of each wave paddle provide additional feedback for 
active wave absorption (reflection compensation). The controller also manages drive signals, implements the active wave absorption algorithms, monitors the health of the drives and manages the drives based on commands received from the Operator's Console.

The controller is built on the Cannon Automata A2 all-in-one programmable automation controller platform. The A2 is based on the Intel Atom processor and has built-in CAN Bus support, digital I/O, gigabit Ethernet and hardware support for real time Ethernet communication with the servo drives. The Akamina real time Linux operating system has been ported to the hardware platform supporting control update rates up to $500 \mathrm{~Hz}$. All position and velocity feedback and control signals transmitted over the Sercos III interface.

The wave generator control system provides support for active wave absorption (reflection compensation) for both unidirectional and multi-directional waves. This is accomplished by measuring the actual wave height along the surface of each wave paddle and comparing it to the expected wave height. The active wave absorption method used by Akamina Technologies uses a velocity controller that tracks the desired velocity and the corresponding desired wave height at the same time. When computing the desired velocity of the wave paddle, the control algorithm looks at the difference between the desired and measured wave height and converts this difference to a velocity correction. The velocity correction is added to the desired velocity which the controller then tracks.

Figure 8 provides additional details on the A2 Controller, the 4 dual-axis Inverters (servo drives) and the interface logic.The A2 Controller and the 4 Inverters are connected on a SERCOS III loop. Real-time data and service channel messages are exchanged between the Controller and the Inverters. Unlike common Ethernet protocols, SERCOS III provides Ethernet with a guaranteed real-time messaging capability.

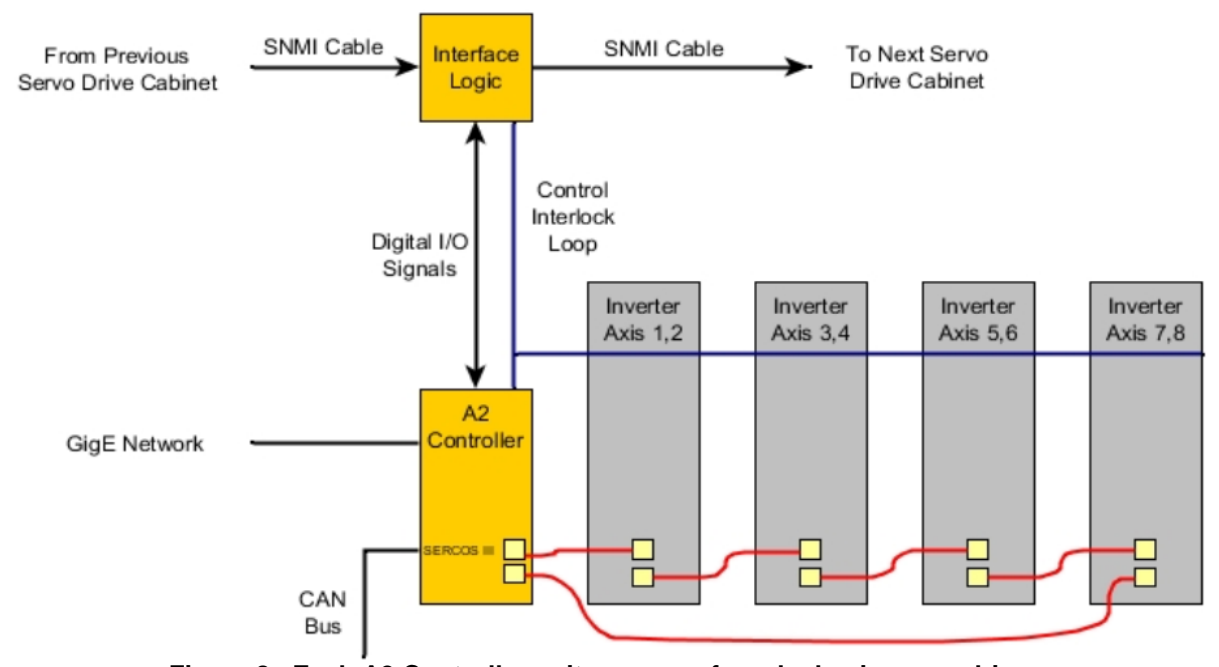

Figure 8. Each A2 Controller unit manages four dual-axis servo drives.

Each Servo Drive Cabinet also includes a custom Interface Logic board. The circuitry on this board provides access to the synchronization and control interlock signals carried in the Synchronization, Network, Management and Interlock cables. Various relays and other components allow the A2 to close its section of the control interlock loop and detect when the control interlock is opened elsewhere in the loop. The synchronization signals guarantee that the real-time messaging loops within each of the Servo Drive Cabinets are synchronized and that wave generation starts and stops at the same instant within all Servo Drive Cabinets.

The CAN Bus interface on the Controller allows the controller to acquire wave height feedback from the Akamina AWP-300 digital wave height gauges mounted on the face of each wave paddle. This information is critical to the active wave absorption system, which works actively absorb waves approaching the wave generator (i.e. waves reflected from a model shoreline or structure).

\section{Wave Generator Module}

As described above, each wave generator module consists of eight $0.41 \mathrm{~m}$ wide by $1.7 \mathrm{~m}$ tall wave paddles and their drivetrains mounted on a rigid steel support frame. The drive system includes an 
electric motor, servo-drive and ball-screw. The drivetrain components have been selected to meet the specified design criteria for wave heights and wave periods. The wave paddle stroke of $1.1 \mathrm{~m}$ and maximum velocity of $1 \mathrm{~m} / \mathrm{s}$ provide the additional stroke and velocity required for simultaneous wave generation and active wave absorption (reflection compensation).

\section{ADVANCED WAVE GENERATION FEATURES}

Several important capabilities have been added to GEDAP and WaveGen-Studio as part of the development efforts in support of this new wave generation system. The capabilities added include:

- $\quad$ software enhancements to improve active wave absorption performance by including diffraction effects when computing the expected wave heights at each wave paddle;

- development of a new corner reflection method based on a linear diffraction model;

- development of second-order wave generation software according to the approach described by Schäffer $(1993,1996)$ and then modified by Schäffer and Steenberg (2003); and

- $\quad$ support for use of a Gaussian directional spreading function in the synthesis of 3D waves.

\section{Active Wave Absorption Improvements}

The algorithm used to implement active wave absorption (AWA) based on feedback from wave gauges mounted on the face of each wave paddle, combined with pre-computed time-histories of the expected wave heights at each of the wave paddles, is based on earlier work reported in Laurich (1994). The control algorithm adjusts the motion of the wave paddles in real time to reduce the difference between the measured water surface elevation and the expected surface elevation. The compensation is effective over a wide range of frequencies. However, past experience with oblique long-crested waves showed that the earlier AWA system led to generation of waves that were larger than desired in certain cases.

The work done to improve active wave absorption performance included using a linear diffraction model to numerically simulate the effects of active wave absorption without considering linear diffraction effects. The wave synthesis software was then extended to include linear diffraction effects with the output of the synthesis software then used in a numerical simulation. These simulations showed that disregarding diffraction effects for oblique long-crested waves can indeed result in wave heights that are larger than expected. Furthermore, these errors are reduced considerably when diffraction effects are included. The AWA system implemented for the new NRC wave generator has been improved by including the effects of diffraction.

The AWA algorithm has also been extended to improve the absorption of waves approaching the machine from different directions. In 3-D mode, water-surface elevation feedback from three adjacent paddles is used to compute the compensation for the central paddle. The information from the adjacent paddles is used to estimate the direction of the incoming wave and compute the appropriate amount of compensation. Less compensation is required for waves that approach obliquely compared with those approaching head on.

\section{Side-wall Reflection Improvements}

Directional wavemakers often have difficulty generating homogenous wave conditions in all parts of a basin due to local effects caused by processes such as wave reflection and diffraction. For certain conditions, the area where a desired seastate can be realized, and hence where testing can/should be conducted, is surprisingly limited. In response, Funke and Miles (1987) and Dalrymple (1989) advanced side-wall reflection methods to expand the area where homogeneous wave conditions are realized. These methods involve intentionally reflecting waves from rigid side-walls located at the end of a wave generator in order to expand the area where desired wave conditions are produced.

A new method of side-wall reflection, reported in Miles (2015), has been developed as part the development behind the new NRC wavemaker. In the new method, a linear diffraction wave model is used to compute the wave paddle positions that result in uniform wave heights at the end of the side walls. Numerical simulations have been conducted to test the new method. These simulations allowed comparisons to be made between the wave fields generated using the standard Snake Principle, and those generated using the side-wall reflection methods of Funke and Miles (1987), Dalrymple (1989), and the new Linear Diffraction method. The study results showed that the new side-wall reflection method based on diffraction modelling performs very well for a wide variety of frequencies, wave angles and sidewall lengths. More importantly, the new method produces a much larger area of uniform wave height in the basin for both regular and irregular waves compared with previous methods. 


\section{Second-Order Wave Generation}

Conventional wave synthesis and generation systems based on first-order (linear) wave theory are unable to properly generate nonlinear waves in shallow water. The use of first-order methods invariably leads to generation of spurious waves at low and high frequencies that propagate as free waves, i.e. not bound to the primary waves as they should be. These free sub- and super-harmonics contaminate the waves realized in a flume or basin, altering wave profiles and kinematics. Erroneous generation of spurious free waves can have significant impacts on important processes, such as wave overtopping and moored ship motions, and lead to erroneous experimental results.

Second-order wave generation is aimed at reducing or eliminating the spurious free waves, leaving only the proper sub- and super-harmonics that travel bound to the primary waves. Support for secondorder wave generation according to the approach described by Schäffer (1996) and then modified by Schäffer and Steenberg (2003) has been developed with the assistance of the Environmental Hydraulics Institute IH Cantabria. IH Cantabria initially developed second-order synthesis methods for regular and irregular waves propagating normally to the wave generator. This method was then extended to support both regular and irregular oblique waves following Schäffer and Steenberg (2003). The methods were validated using numerical simulations for both regular and irregular waves travelling perpendicularly to the wave paddle. Subsequent physical model tests carried out in a 2D wave flume and a 3D facility confirmed that the new methods performed as expected. The resulting implementation was then integrated into GEDAP for use in the new system developed for the NRC.

The effectiveness of the second-order wave generation method for the case of bi-chromatic waves is illustrated in Figure 9. In this figure, the primary waves are shown in blue while the sub-harmonic waves, isolated by low-pass filtering, are shown in red, amplified by a factor of ten for enhanced clarity. When first-order theory is used (Figure 9a), the resulting low-frequency waves are much smaller in amplitude and the set-down (the periodic troughs of the low-frequency wave train) is not centered on the wave groups, as it should be. With first-order generation, the measured set-down also varies with distance as the waves propagate away from the wave generator. As shown in Figure 9b, the use of second-order theory corrects these deficiencies.

a) First-order:

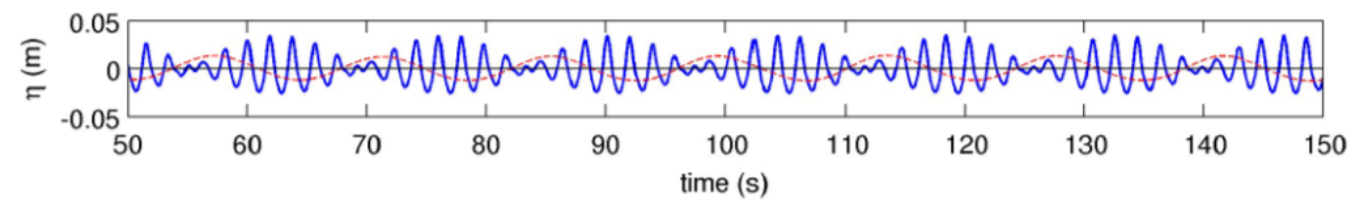

b) Second-order:

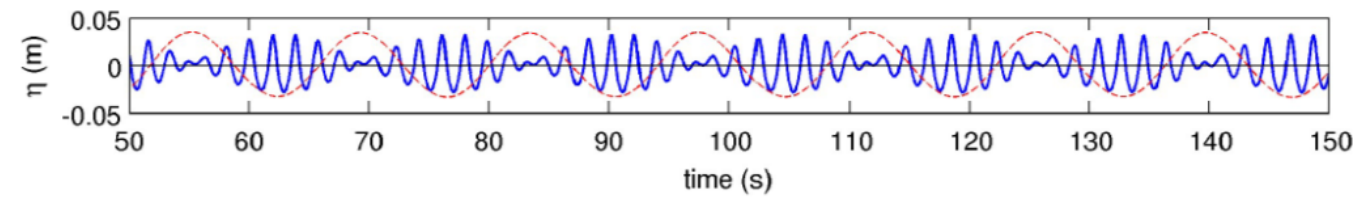

Figure 9. Comparison of bi-chromatic waves synthesized and generated using a) first-order and b) secondorder theory. The low-frequency waves, shown in red, are amplified 10x for greater clarity.

\section{Gaussian Spreading Function}

Previous versions of GEDAP and WaveGen-Studio provided support for synthesis of directional spreading functions and multidirectional wave fields wherein the distribution of energy with direction followed a cosine-power form. A new spreading function formulation has been added to WaveGenStudio wherein the distribution of wave energy with direction follows a Gaussian distribution. The Gaussian spreading function is defined by the mean wave angle and the spreading width. The theoretical spreading function is normalized so that it has a unit area as it is a probability density function. 


\section{COMISSIONING}

The new wave generator was commissioned at NRC's Ottawa labs in May 2016. A set of commissioning trials involving generation and measurement of regular waves, bi-chromatic waves, irregular long-crested and short-crested wave fields at two water depths was conducted to demonstrate the capabilities of the new system. A second set of trials was conducted in January 2017 to confirm the performance of the new second-order wave generation and active wave absorption capabilities. The ability to move the modules within the $50 \mathrm{~m}$ x $30 \mathrm{~m}$ basin quickly and safely using a forklift (lift truck) was also confirmed, as was the ability to divide the modules into two shorter wavemakers.

The results of these trials confirmed that the new wave generator is able to generate a wide range of wave conditions with excellent fidelity and repeatability. The ability of the active wave absorption (reflection compensation) algorithm to absorb 96\% of incident wave energy over a broad range of frequencies and directions was confirmed. Two wave height performance curves for irregular waves (long-crested or short-crested) with mean direction normal to the paddles is presented in Figure 10. For $1.0 \mathrm{~m}$ water depth the new wavemaker is able to generate irregular waves with significant wave heights greater than $0.4 \mathrm{~m}$ for peak wave periods between $\sim 2.3 \mathrm{~s}$ and $\sim 3.2 \mathrm{~s}$. At shorter periods (higher frequency) the wave height is limited by wave steepness, whereas available paddle stroke is the limiting factor at larger periods (lower frequencies). For $0.6 \mathrm{~m}$ water depth, the maximum significant wave height that can be generated exceeds $0.3 \mathrm{~m}$, and is limited by depth-limited wave breaking in addition to wave steepness at higher frequencies and paddle stroke at lower frequencies.

It should be noted that for waves generated in oblique directions (not normal to the wave paddles) when the new side-wall reflection method is used to expand the area over which homogeneous wave conditions are produced, the maximum wave heights that can be generated are roughly $20 \%$ smaller than shown in Figure 10. This reduction in wave height capability is due to the extra paddle stroke demanded by the side-wall reflection method. The upper wave height limit is also reduced when the active wave absorption (reflection compensation) algorithm is engaged, again due to the additional demands on paddle stroke required to absorb incoming waves while generating.

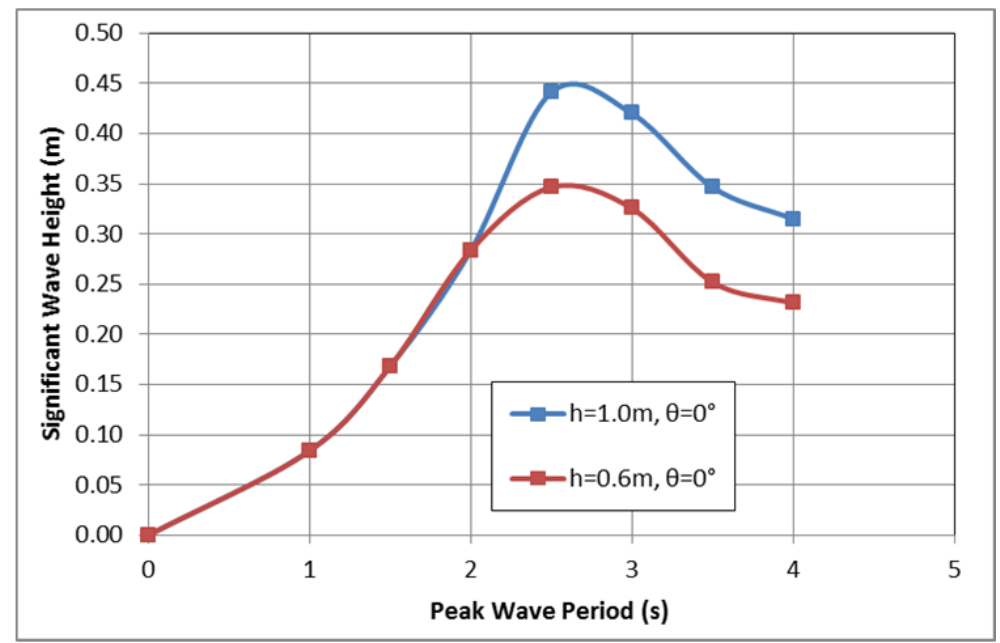

Figure 10. Performance curves for irregular waves.

\section{CONCLUSIONS}

New wave generation systems with improved capabilities for generating realistic wave conditions in test facilities at larger scales are essential to increase the quality of coastal engineering research and practice. Towards this end, a new multidirectional wave generator that is powerful, modular and portable has been designed, built and commissioned for use in a new $50 \mathrm{~m}$ by $30 \mathrm{~m}$ wave basin at the National Research Council (NRC) labs in Ottawa, Canada. The new wave generator is comprised of nine portable modules, each supporting eight $0.41 \mathrm{~m}$ wide by $1.7 \mathrm{~m}$ tall wave paddles. Each wave paddle has a stroke of $\pm 0.55 \mathrm{~m}$ combined with a rated maximum velocity of $1.0 \mathrm{~m} / \mathrm{s}$, and is powered by a synchronous servo motor acting through a backlash-free ball-screw. Several modules can be joined together using specially designed clamps to form integrated wavemakers of various lengths. The new 
wave generator can generate short-crested and long-crested irregular waves with significant wave heights exceeding $0.4 \mathrm{~m}$, and waves with periods from $0.5 \mathrm{~s}$ to $5 \mathrm{~s}$. The nine modules can be arranged in various configurations: to form a single $29.5 \mathrm{~m}$ long wave machine at any location in the $50 \mathrm{~m}$ by $30 \mathrm{~m}$ basin; to form an "L" configuration along two adjacent walls; or even to form two or more wave machines separated from each other, having a combined length of $29.5 \mathrm{~m}$. Innovative design has been combined with lightweight materials and aerospace construction methods to achieve a very high rigidity/strength-to-weight ratio that delivers improved frequency response and very precise control over wave paddle motions. Regenerative power supplies have been used to significantly reduce energy consumption and operational cost. The total weight of each module was kept below 2,350 kg to ensure that it could be moved easily within the basin using only a forklift or hydraulic lift truck. For stability when generating large waves, the modules can either be fastened to the basin floor or stabilized by adding ballasting weights on top of the steel support frame. This innovative and capable new machine was developed for the National Research Council of Canada by Akamina Technologies (Canada) in partnership with +D (Spain) with support from Respect Industries (Canada), Bosch-Rexroth (Canada), IH Cantabria (Spain) and M.D. Miles (Canada).

The new wave generator features state-of-the-art software and control systems, including enhanced capabilities for active wave absorption (2D and quasi-3D modes), second-order wave generation (for accurate generation of sub- and super-harmonics), side-wall reflection (to expand the area where homogenous wave conditions are generated), and more. The new wave generator is operated and controlled through the WaveGen-Studio software - a powerful, feature-rich system designed for managing hydrodynamic experiments and physical model studies. WaveGen-Studio combines functionality for data acquisition, wave synthesis, wave generation and wave data analysis in a seamless, intuitive graphical interface designed to allow users to easily manage even the most complex experiments. The wave synthesis, wave data analysis and plotting functionality within WaveGenStudio are based on the GEDAP software system developed and proven over several decades at the NRC.

Other noteworthy features not already mentioned include:

- a control system that accurately tracks desired wave paddle position or velocity drive signals with minimal phase lag and negligible attenuation over a wide range of frequencies,

- a dynamic calibration procedure that allows the electro-mechanical transfer functions of the individual segments to be computed and used to pre-compensate for the dynamic response of each segment,

- remote monitoring and management of individual servo drives from the Operator's Console,

- control by wire - all feedback and control signals are acquired over a communications network,

- $\quad$ advanced safety features including safety lock-outs plus audible and visual warnings. 


\section{ACKNOWLEDGEMENT}

The authors wish to thank all reviewers of this paper for providing constructive feedback on early

drafts of the manuscript. The outstanding contributions of the many engineers and technicians who were involved in designing and fabricating the new wave generator are hereby acknowledged with immense gratitude.

\section{REFERENCES}

Cornett, A.M., Miles, M.D., 1991. Simulation of Hurricane Seas in a Multidirectional Wave Basin, $J$. Offshore Mechanics and Arctic Eng., 113:219-227.

Cornett, A.M., Miles, M.D., Pelletier, D., 1995. Measurement and Analysis of Multidirectional Waves Using Free Surface Slopes. Proc. $5^{\text {th }}$ Int. Sym.On Wave Measurement and Analysis (Waves'95), Madrid, Spain.

Dalrymple, R.A., 1989. Directional Wavemaker Theory with Sidewall Reflection. J. Hydraulic Research, 27,1:23-34.

Funke, E.R., Miles, M.D., 1987. Multidirectional Wave Generation with Corner Reflectors, NRC Report TR-HY-021, Ottawa, Canada.

Laurich, P.H., 1994. Active Wave Absorption Using Feedback From a Wave Probe Attached to the Wave Board. NRC Controlled Technical Report IECE-CEP-CTR-012, Ottawa, Canada.

Mansard, E. and Miles, M., 2010. Laboratory Simulation of Waves, in Handbook of Coastal and Ocean Engineering. World Scientific Publishing, pp 1107-1134.

Miles, M.D., 1989. User Guide for GEDAP Version 2.0 Wave Generation Software. NRC Report LMHY-034, Ottawa, Canada.

Miles, M.D., 1997. GEDAP Users Guide for Windows. NRC Report HYD-TR-021, Ottawa, Canada.

Miles, M.D., 2015. A New Sidewall Reflection Method for Generation of Directional Waves, Report for Akamina Technologies Ltd.

Mitsuyasu, H., 1972. The One-Dimensional Wave Spectra at Limited Fetch. Proc. $13^{\text {th }}$ Int. Conf. on Coastal Engineering.

Nwogu, O., Mansard, E., Miles, M., Isaacson, M., 1987. Estimation of Directional Wave Spectra by the Maximum Entropy Method. Proc. IAHR Seminar on Wave Analysis and Generation in Laboratory Basins, $22^{\text {nd }}$ IAHR Congress, Lausanne, Switzerland, pp. 363-376.

Schäffer, H.A., 1993. Laboratory Wave Generation Correct to Second-Order, Proc. Wave Kinematics and Environmental Forces. Society for Underwater Technologies, London, 29:115-139.

Schäffer, H.A., 1996. Second-Order Wavemaker Theory for Irregular Waves, Ocean Engineering, 23,1:47-88.

Schäffer, H.A., Steenberg, C.M., 2003. Second-Order Wavemaker Theory for Multidirectional Waves, Ocean Engineering, 30:1203-1231. 